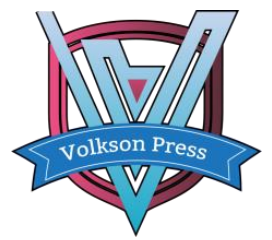

Contents List available at VOLKSON PRESS

Intelligent Computing and Information Engineering (ICIE )

DOI : http://doi.org/10.26480/icie.01.2017.17.19

Journal Homepage: : https://www.intelcomp-design.com/

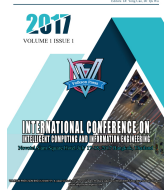

\title{
Quantitative Comparative Analysis of Multilayer Method, Real Space Method and Bloch Wave Method in Analog Circuit Calculation
}

\author{
WANG Tao ${ }^{1}$, SUN Wen ${ }^{2, *}$ \\ ${ }^{1}$ Xianning vocational technical college, Xianning, China \\ ${ }^{2}$ Tellhow Polytron Technologies Inc, Shanghai, China \\ *zhaoleies@163.com
}

This is an open access article distributed under the Creative Commons Attribution License, which permits unrestricted use, distribution, and reproduction in any medium, provided the original work is properly cited

\section{ARTICLE DETAILS}

\section{Article History:}

Received 12 May2017

Accepted 12 July 2017

Available online 14 September 2017

Keywords:

Electron diffraction, Bloch wave method, Multilayer method, Real space method.

\section{ABSTRACT}

The electron diffraction calculation is one of the most important methods of electron crystallography. It is very important to select the method of electron diffraction calculation for the study of electronic crystals. At present, there are mainly three kinds of calculation methods for electron diffraction. They are sorted into Bloch wave method, multilayer method and real space method from the time sequence of occurrence. The three methods are based on different principles, so the advantages of the three methods cannot be obtained in the comparative analysis. In this paper, these three methods of calculation are rewritten as a unified expression. After the specific case analysis, we can easily come to conclusion about the advantages concerning the three methods among the accuracy, speed and applicability of the three aspects.

\section{Introduction}

The Bloch wave method, also called the eigenvalue method, was proposed by Bethe in 1928. Due to rigorous theory, The Bloch wave method is well received [1]. As the most traditional method of resolving the Schrodinger equation, the Bloch wave method is widely used to determine lattice constants of materials, accurate determination of structural factors, electronic energy loss spectra and so on in analog calculations [2]. Because of the diagonalization of the matrix in the Bloch wave method, it will take more time for CPU to run. In order to solve the trouble, Cowley and Moodie proposed the multilayer method in 1957 [3]. Inspired by physical optics, Multilayer method is different from Schrodinger equation. It belongs to an approximate meth od. Because the calculation model of multilayer method is projection potential instead of continuous type, the propagation of wave function is separated from diffraction. Taking into account these circumstances, Van Dyck proposed the real space method. The last way is real space method [4]. Based on the Schrodinger equation, the calculation theory is more rigorous. Meanwhile, all the calculations are based on real space. However, we face the fact that the Real space calculation method is slower to calculate. Therefore, the theory is not widely used in electron diffraction calculation. Based on the case of $\mathrm{Mg} \mathrm{Al}_{2} \mathrm{O}_{4}$ crystal, this paper analyzes the advantages of three methods in terms of accuracy, speed of calculation and applicability.

\section{REWRITING OF A UNIFIED EXPRESSION}

Multi-layer method, real space method and Bloch wave method. Although these three methods are based on the principle is not the same, real space method and Bloch wave method are based on the fixed Schrodinger equation, and multi-layer method based on physical optics. However, the three methods can be written as a unified Schrodinger equation analytical expression. As shown in the following formula.

$$
\varphi(r)=\exp \left[\frac{i z}{4 \pi k_{s}}\left(\Delta_{x y}+2 k_{x y} \nabla_{x y}+4 \pi^{2} U(\bar{r})\right)\right] \varphi(0)^{o} .
$$

The computational requirements of the three computational methods are quite different. Real space method for calculating wave function $\varphi(r)$ in positive space, the multilayer method calculates the wave functions in both the positive and the inverted spaces, the Bloch wave method calculates the wave function in the inverted space after diagonalization of the matrix. In order to satisfy the difference between the three calculation methods in the Analog calculation, the formula 1 is modified.

The modified expression of the multilayer method is shown in Equation 2 below.

$$
\varphi\left(x, y, z_{j+1}\right)=\exp \left[\frac{i \varepsilon_{j}}{4 \pi k_{s}}\left(\Delta_{x y}+2 k_{x y} \nabla_{x y}\right)\right] \cdot \exp \left[\frac{i \varepsilon_{j}}{4 \pi k_{s}} 4 \pi^{2} U(\vec{r})\right] \varphi\left(x, y, z_{j}\right)^{o} .
$$

In the formula, $\varepsilon_{j}$ represents the thickness of the j-th layer.

For the real space method, expression is rewritten as follows 3,4 . 


$$
\begin{aligned}
& \varphi\left(x, y, z_{j+1}\right)=\exp \left[\frac{i \varepsilon_{j}}{4 \pi k_{s}}\left(\Delta_{x y}+2 \vec{k}_{x y} \nabla_{x y}+4 \pi^{2} U(\vec{r})\right)\right] \varphi\left(x, y, z_{j}\right)^{o} . \\
& \varphi\left(x, y, z_{j+1}\right)=\sum_{n=0}^{p} \frac{1}{n !}\left[\frac{i \varepsilon_{j}}{4 \pi k_{s}}\left(\Delta_{x y}+2 \vec{k}_{x y} \nabla_{x y}+4 \pi^{2} U(\vec{r})\right)\right]^{n} \varphi\left(x, y, z_{j}\right)^{o} .
\end{aligned}
$$

In the formula, $\varepsilon_{j}$ represents the thickness of the j-th layer. This expression needs to be solved by iterative methods. For the Bloch wave method, the modified Schrodinger equation expression is shown in Equation 5 below.

$\varphi(x, y, z)=\exp \left(\frac{i z}{4 \pi k_{s}} A\right) \phi(x, y, 0)=\exp \left[\frac{i z}{4 \pi k_{s}}(\tilde{S} L S)\right] \varphi(x, y, 0)=\tilde{S} \exp \left(\frac{i z}{4 \pi k_{s}} L\right) S \varphi(x, y, 0)$

In the formula, the matrix A is the scattering matrix. Since the matrix A contains diagonal elements and non-diagonal elements, it needs to be orthogonally normalized for solving. $\mathrm{L}$ is a diagonal matrix, $\mathrm{S}$ is an ortho normalized matrix, and $\mathrm{Z}$ is the thickness of the sample. The Bloch wave method requires stratification of the sample.

\section{MG $\mathrm{AL}_{2} \mathrm{O}_{4}$ CRYSTAL EXAMPLE CALCULATION RESULTS}

In order to quantitatively compare the three methods in the accuracy, speed and applicability of the three aspects of the advantages, the example of Mg $\mathrm{Al}_{2} \mathrm{O}_{4}$ crystal is analyzed and three methods are used to calculate the wave function of the outgoing surface of crystal. The results are shown below.

The $\mathrm{Mg} \mathrm{Al}_{2} \mathrm{O}_{4}$ crystal is a face centered cube structure with a lattice parameter of $a=b=c=0.808 n m, \alpha, \beta, \gamma$ are at right angles, the space group is $F d \overline{3} m$ What's more, a single cell has 64 atoms. There are the necessary dates: the simulated parameters are the incident wavelength of $0.0032 \mathrm{~nm}$, the sample thickness of $\mathrm{Z}$ is $12.0 \mathrm{~nm}$, and the incident electron beam incident along the [001] direction. Also, the pixel size is a/80, $\mathrm{b} / 80$. The calculation method results are shown in table 1,2 and 3 .

\begin{tabular}{|c|c|c|c|c|c|c|c|c|c|}
\hline \multirow{2}{*}{\multicolumn{2}{|c|}{$\begin{array}{l}\text { thickness } \\
\text { Method Pixel Exror }\end{array}$}} & \multicolumn{2}{|l|}{5.0} & \multicolumn{2}{|l|}{3.0} & \multicolumn{2}{|l|}{1.0} & \multicolumn{2}{|l|}{0.7} \\
\hline & & Amplitude & Intensity & Amplitude & Intensity & Amplitude & Intensity & Amplitude & Intensity \\
\hline RS-BW & $160 \times 160$ & $4.2178 \mathrm{E}-04$ & $2.1987 \mathrm{E}-07$ & $4.1789 \mathrm{E}-04$ & $2.1278 \mathrm{E}-07$ & $4.2178 \mathrm{E}-04$ & $2.1268 \mathrm{E}-07$ & $4.2178 \mathrm{E}-04$ & $2.1278 \mathrm{E}-07$ \\
\hline MS-BW & $160 \times 160$ & $1.3189 \mathrm{E}-01$ & $1.4189 \mathrm{E}-02$ & $2.9268 \mathrm{E}-02$ & $1.3189 \mathrm{E}-03$ & $8.3168 \mathrm{E}-03$ & $2.0267-04$ & 4.0157E-03 & $4.9937 \mathrm{E}-05$ \\
\hline
\end{tabular}

Table 1: Three calculation errors of $\mathrm{Mg} \mathrm{Al}_{2} \mathrm{O}_{4}$ crystals

Table 2: Simulation of time and error for different numbers of diffraction beams

\begin{tabular}{llll}
\hline \multirow{2}{*}{$\begin{array}{l}\text { Number of Diffraction } \\
\text { Beam }\end{array}$} & \multicolumn{2}{l}{ Error of intensity } & \multirow{2}{*}{ Time[s] } \\
\cline { 2 - 3 } & Non-revision & Revision & \\
\hline 53 & $5.0230 \mathrm{E}-02$ & $9.0473 \mathrm{E}-03$ & 0.07 \\
67 & $3.0121 \mathrm{E}-02$ & $4.2315 \mathrm{E}-03$ & 0.09 \\
95 & $1.3926 \mathrm{E}-02$ & $1.5128 \mathrm{E}-04$ & 0.13 \\
125 & $9.1379 \mathrm{E}-03$ & $5.6289 \mathrm{E}-04$ & 0.19 \\
155 & $5.4167 \mathrm{E}-03$ & $3.0158 \mathrm{E}-04$ & 0.52 \\
231 & $2.5289 \mathrm{E}-03$ & $1.2028 \mathrm{E}-04$ & 1.21 \\
291 & $1.27981 \mathrm{E}-3$ & $3.82579 \mathrm{E}-5$ & 2.35 \\
800 & Criterion & Criterion & 57.02 \\
\hline
\end{tabular}

\begin{tabular}{|c|c|c|c|c|}
\hline $\begin{array}{l}\text { Slice thickness }[\mathrm{nm}] \\
\quad \text { Method }\end{array}$ & 0.5 & 0.3 & 0.1 & 0.04 \\
\hline BW & 2.420 & 2.420 & 2.420 & 2.420 \\
\hline RS & 0.187 & 0.187 & 0.187 & 0.187 \\
\hline MS & 0.031 & 0.062 & 0.099 & 0.201 \\
\hline
\end{tabular}

Table 3: Three simulation calculation time comparison table

\section{COMPARISON OF THREE CALCULATION METHODS}

Generally, the quality evaluation of a simulation calculation method is composed of three aspects, the accuracy of the results, the computation time and speed, the applicability of the method. The measurement accuracy of the experimental data determines the accuracy of the calculation results. At the same time, the speed of analog computing depends on the computer's ability to solve and the complexity of the computational model. Therefore, this section evaluates the advantages and disadvantages of the three methods through the above three evaluation directions.

\subsection{Accuracy comparison analysis}

The accuracy analysis of the calculation is mainly reflected in the error of the calculation results. From the 4 and 5 formula, we can see that the real space method is in the positive space, while the Bloch wave method is in the inverted space. As the pixel size is not infinitely small, therefore, in the simulation calculation, the numerical differential calculation results will cause some error. At the same time, the Bloch wave method also has some errors in the diagonalization of the non-diagonal elements. Both errors of Diagonalization and pixel size are concerning numerical value. Compared with the pixel size error, diagonalization of the error is relatively smaller. At the same time, the error caused by Bloch wave method is much smaller than the error of real space. Therefore, based on the simulation results of Bloch wave method, the errors of the real space method and the multilayer space method are analyzed and compared.

Analysis of the data in Table 1 shows that in the same pixel size, the error of the real space method is very small. Compared with the real space method, the multilayer space method has a large relative error, and increases with the thickness of the slice. 
real space method, multi-layer space method.

\subsection{The calculation speed of comparative analysis}

The time required for the simulation is related to the branching beam of the crystal. $\mathrm{Mg} \mathrm{Al}_{2} \mathrm{O}_{4}$ crystals have 800 scattering beams. The time spent in the Bloch wave method is to diagonalize the matrix. It can be seen from the literature that the calculation time of the diagonalization of the matrix is positively proportional to the three party of the diffracted beam number. And the use of bite potential can save the Bloch wave method simulation time.

It can be seen from Table 2 that the total calculation time of the Bloch Bobby method is much longer than that of the real space method. Even after the use of Baye potential to accelerate, the computation time is much longer than the $0.187 \mathrm{~s}$ of the real space method.

Analysis Table 3 shows, The $\mathrm{Mg} \mathrm{Al}_{2} \mathrm{O}_{4}$ crystal is in the thickness of $12.0 \mathrm{~nm}$ and the incident electron beam is in the direction of [001], when the pixel sizes are a / 80 and b / 80, by the method of multilayer FFT transform, the corresponding slice thicknesses are $0.5 \mathrm{~nm}, 0.3 \mathrm{~nm}, 0.1 \mathrm{~nm}$, and $0.04 \mathrm{~nm}$, respectively, and their simulated computation time is $0.031 \mathrm{~s}, 0.062 \mathrm{~s}, 0.099 \mathrm{~s}, 0.201 \mathrm{~s}$. From the table we can see that the calculation time is almost. However, the multilayer method belongs to the approximate method, and its accuracy is lower than that of the real space method. When the slice thickness is $0.5 \mathrm{~nm}, 0.3 \mathrm{~nm}$ and 0.1 $\mathrm{nm}$, the computation time of the multilayer method is shorter than the real space method. But when the slice thickness is $0.04 \mathrm{~nm}$, there is little difference between the multi-layer method and the real space method. Therefore, the real space method is more flexible than the multilayer method, and is a better method for simulation calculation

\subsection{Comparative analysis of applicability}

Applicability comparison is to summarize the scope of application of the three methods. Bloch wave method is generally only applicable to the complete crystal structure. However, the multilayer method and the real space method apply to complete crystal and defective crystals. Thus, the multi-layer method and the real space method are more extensive than the Bloch wave method.

\section{SUMMARY}

In order to compare the advantages and disadvantages of three methods, namely multi-layer method, real space method and Bloch wave method. In this paper, the expression of the three methods is rewritten into the unified equation. Taking the $\mathrm{Mg} \mathrm{Al}_{2} \mathrm{O}_{4}$ crystal with face centered cubic structure as an example, the quantitative analysis can be drawn as follows.

The computational accuracy of the Bloch wave method is the highest, while the multilayer space method is the lowest precision, belonging to the approximation method. In terms of computation time, the Bloch wave method has the longest time while the multilayer space method is the shortest. In addition, the multilayer method and the real space method have the most extensive applicability. Correspondingly, the Bloch wave method is only suitable for the complete crystal structure.

\section{REFERENCES}

[1] Coene, W., Dyck, D.V. 1984. The real space method for dynamical electron diffraction calculations in high resolution electron microscopy. III. A computational algorithm for the electron propagation with its practical applications, Ultramicroscopy 15, 287-300.

[2] Coene, W., Dyck, D.V. 1984. The real space method for dynamical electron diffraction calculations in high resolut ion electron microscopy. II. Critical analysis of the dependency on the input paramters, Ultra microscopy $15,41-50$.

[3] Sang, X., Kulovits, A., Wiezorek, J. 2013. Comparison of convergent beam electron diffraction methods for simultaneous structure and Debye Waller factor determination. Ultramicroscopy, 126, 48-59.

[4] Dyck, D.V., Coene, W. 1984. The real space method for dynamical electron diffraction calculations in high resolution electron microscopy. I. Principles of the method, Ultra microscopy 15, 29-40. 